\title{
Analysis of the Flow Mobility of Multimedia Traffic across Software Defined Networks
}

\author{
Ronnie D. Caytiles and Sunguk Lee* \\ Department of Multimedia Engineering, Hannam University \\ 133 Ojeong-dong, Daeduk-gu, Daejeon, Korea \\ rdcaytiles@gmail.com,*sulee0612@hnu.kr
}

\begin{abstract}
Recently, the Software Defined Networking (SDN) architecture has been transforming the traditional networking infrastructures in order to meet the requirements of today's businesses, enterprises, telecommunication carriers, and end users. This is in response to the overflowing amount of multimedia contents wherein the availability is continuously increasing over the Internet. The SDN utilization addresses the ineffectiveness of the centralized and distributed mobility management solutions enabling the flow mobility of multimedia traffic become programmable and dynamic. This paper deals with the analysis of the flow mobility of multimedia traffic across software defined Networks. It aims to provide an efficient mobility management support for handovers of mobile devices in heterogeneous wireless networks.
\end{abstract}

Keywords: flow mobility, software defined networking (SDN), SDN Controller, centralized mobility management

\section{Introduction}

The volume of multimedia traffic available on the Internet is continuously growing as telecommunication technologies are evolving. In 5 years, it is projected that this multimedia traffic will be tripled based on the complete visual networking index (VNI) forecast [1] held in the year 2016. Thus, multimedia traffic congestion is expected to overwhelm the limited network resources without proper orchestration and mobility management support. In addition, the current trends of mobile devices evolution, server virtualization, and the advent of cloud computing services have driven the networking system operators to re-evaluate the existing traditional network architectures. The traditional network architectures are generally hierarchical wherein its operators mostly considers centralized mobility management solutions in supporting handovers of mobile devices across the heterogeneous wireless networks. They are often built with layers of Ethernet switches and routers that are organized like a tree structure.

The centralized mobility management solutions were successful when utilized in a client-server computing environment. All of the multimedia traffic in these mobility management solutions are controlled and managed by a central mobility anchor. In addition, the multimedia traffic needs to traverse the central mobility anchor that creates bottleneck and congestion issues, suboptimal routing, low scalability, and other related challenges [2, 3]. Moreover, with the current requirements of industries, business enterprises, and organizations, the centralized mobility solutions can be insufficient to address their needs. Thus, alternative novel and dynamic mobility management solutions will be required in order to provide

Received (July 27, 2017), Review Result (October 25, 2017), Accepted (October 30, 2017)

* Corresponding Author 
balanced flow mobility specifically on real-time multimedia services across the heterogeneous wireless networks.

This paper aims to provide an analysis of the flow mobility of multimedia traffic across software defined networks. The programmable features of SDN are utilized to provide mobility management support for handling handovers of mobile devices that are continuously roaming over heterogeneous wireless networks. It decouples the data plane that optimizes the routing of multimedia traffic over network entities from the control plane that remains centralized in orchestrating the management of network resources. The functionalities of the SDN controller are critical to the management of flow mobility of multimedia traffic as well as with handling the handovers of mobile devices in the heterogeneous wireless network environment.

The rest of this paper is organized as follows: Section 2 provides a discussion of some motivations on the need to adapt the SDN paradigm as mobility management solution suited for today's computing trends; the overview of the SDN architecture is analyzed in Section 3; the SDN Controller components and functions are identified in Section 4; the flow mobility in SDN is analyzed in Section 5; and the concluding remarks in Section 6.

\section{Motivations}

The following technological trends are continuously evolving that motivates the need to shift into new network paradigm of SDN mobility solutions:

- The resilient proliferation of mobile devices such as smartphones, notebooks, tablets, personal digital assistants (PDAs) employed by increasing number of mobile users have required a continuous and real-time access to network resources. New computing systems specifically on the networking architectures deemed to suffer the pressure in order to accommodate these needs that traditional network systems cannot offer.

- The increasing multimedia traffic has changed the traffic patterns on data centers. The traditional client-server applications can no longer accommodate the current trend of accessing information and multimedia services. In a client-server paradigm, multiple users access multimedia services through a single server. However, current mobile users access multiple servers and databases. Multimedia traffic are traversing multiple paths, routed through several data centers, pushed back and forth across the heterogeneous network environments, before they could be delivered to the mobile users who themselves are continuously changing their point of attachments (PoAs) creating an anytime, anywhere, and any device environment.

- The "Big data" [4] computing has evolved lately and requires not only amount of storage but also additional network capacity and bandwidth. It requires a massive parallel and exceptional processing on numerous servers and data centers communicating with each other. New network architectures require scaling, and communication between heterogeneous systems is a must.

- The rise of Cloud computing [5] has delivered enormous amount of services that requires a dynamic, agile, and large scale network systems. Traditional network architectures can never accommodate the increasing development of such technological development. It requires self-service provisioning, expandable computing scale, storage, as well as network resources. 


\section{Software Defined Networking}

The Software Defined Networking (SDN) refers to a mobility management paradigm that is spearheaded by the Open Networking Foundation (ONF) aimed to address the ineffectiveness of the traditional network architectures $[6,7,8]$. The SDN architecture separates the control and data planes to provide dynamic and flexible network architecture. In SDN, the underlying network infrastructure is abstracted from the applications and leaves the network intelligence being logically centralized [7]. Thus, it has provided the network systems with an exceptional programmability, automation, and network control management that leads to an optimized and efficient mobility management in heterogeneous wireless networks.

The use of SDN architecture for mobility management of network systems can provide the following advantages [8]:

- Directly programmable: Network control is separated from data forwarding functions making is directly programmable.

- Agile: The network-wide multimedia traffic flow is dynamically adjusted through abstraction of forwarding control.

- Centrally managed: Software-based SDN controllers centrally maintain network resources through a single logical switch.

- Programmatically configured: SDN allows network administrators to easily configure and manage network system resources through a dynamic and automated SDN programs.

- Open standards-based and vendor-neutral: Network design and operation is simplified through SDN open standards.

The SDN aims to provide open interfaces that allow the programmability of the control on the mobility management of network resources as well as to the management of the traffic flow in heterogeneous wireless networks. The basic architecture of SDN is shown in Figure 1. It is initially comprised of three planes: the application, control, and data planes [7,8].

- Application plane. The SDN applications (e.g., Business applications) communicate their network requirements towards the Control plane through northbound interfaces (NBIs).

- Control plane. The control plane is comprised of a set of SDN controllers intended to control a set of resources on one or more NEs. The SDN controller provides services to the SDN applications and controls the operations of the NEs in the Data plane. It is responsible for the allocation of multimedia traffic for the optimization of its flow among NEs.

- Data plane. The data plane is comprised of a set of one or more network elements (NEs) and is controlled by the SDN controller through southbound interfaces (i.e., Control-Data Plane Interface (CDPI) agent). It is responsible for multimedia traffic forwarding and processing functions and includes a part of the control and management functions. 


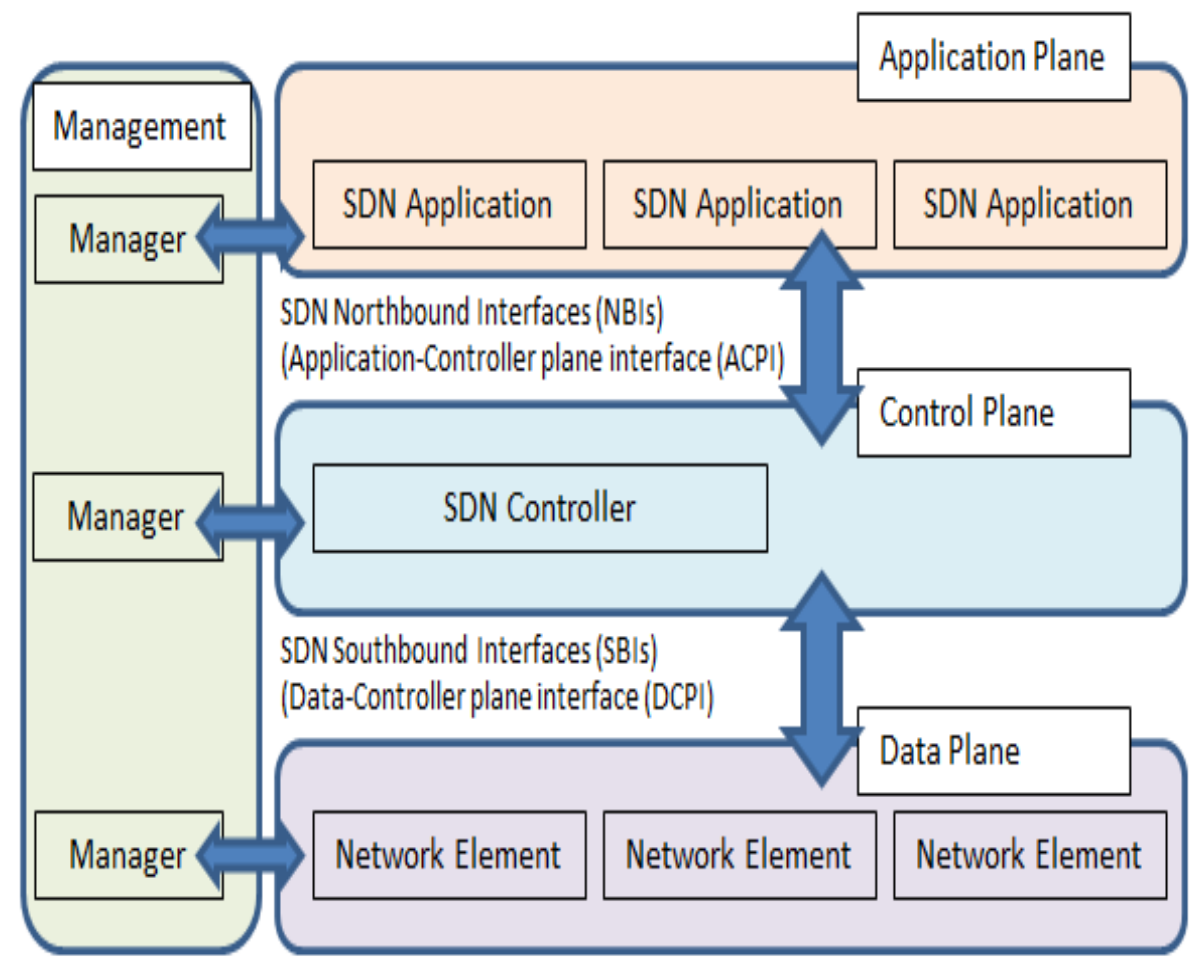

Figure 1. Overview of the Basic Software-Defined Networking Architecture

Figure 1 defines the basic functions, interfaces and components of the SDN architecture. A management function can be added which is essential for the initial configuration of the NEs, and the SDN controller. It is also required for the configuration of the policies that governs the scope of control given to the SDN application and for monitoring the performance of the entire system. In general, the overall management for assigning each NE with its own SDN controller is provided by the management function as well as setting up the configuration policies between SDN controller and applications [8].

The interface between the SDN controller and application plane is referred to as the Northbound Interfaces (NBIs) and between the SDN controller and data plane as Southbound Interfaces (SBIs) [8]. The SDN controller, network elements, and every application are provided with a functional interface to a manager. The manager is responsible for allocating resources from a lower plane towards an entity in the higher plane. The implementation of SDN reflects the separation of the network system that directs the flow multimedia traffic (i.e., where to send the multimedia IP traffic) which is referred to as the SDN controller and the underlying systems (network elements) that is responsible for forwarding the multimedia traffic to the designated mobile device which is referred to as the data plane.

\section{The SDN Controller}

The control plane in SDN can be composed of one or more SDN controllers. The SDN controller is logically centralized that is able to manage more than one NE from the data plane. It orchestrates the interrelated resources and manages the network elements as specified by the SDN application to which the SDN controller is serving. For example, in Figure 2, an SDN application in the application plane is assigned an SDN controller in order to manage the multimedia traffic flow across the network elements in the data plane. SDN controller is also responsible for the 
path computation of multimedia traffic, thus, it is aware of the topology of the NEs that is under its coverage [8].

The SDN controller is viewed as a black box that means that the internal components may vary and is defined through its external behavior. The components of the SDN controller may include the following [8]:

- The Coordinator refers to a functional component of the SDN controller that provides an interface to the Manager in order to setup both the client and server environments.

- The master resource data base (MRDB) maintains the database of all resources that the SDN controller is orchestrating.

- An agent is a functional component of the SDN controller that represents the client's resources and capabilities in the server's environment. The SDN application in the applications plane communicates with the SDN controller through a controlled entity called the agent via the application-control plane interface (ACPI).

- The SDN control logic performs mostly the control and orchestration functions of the SDN controller. SDN controller may able to span multiple NEs through the SDN control logic via the data-control plane interface (DCPI). It also provides the virtualization function.

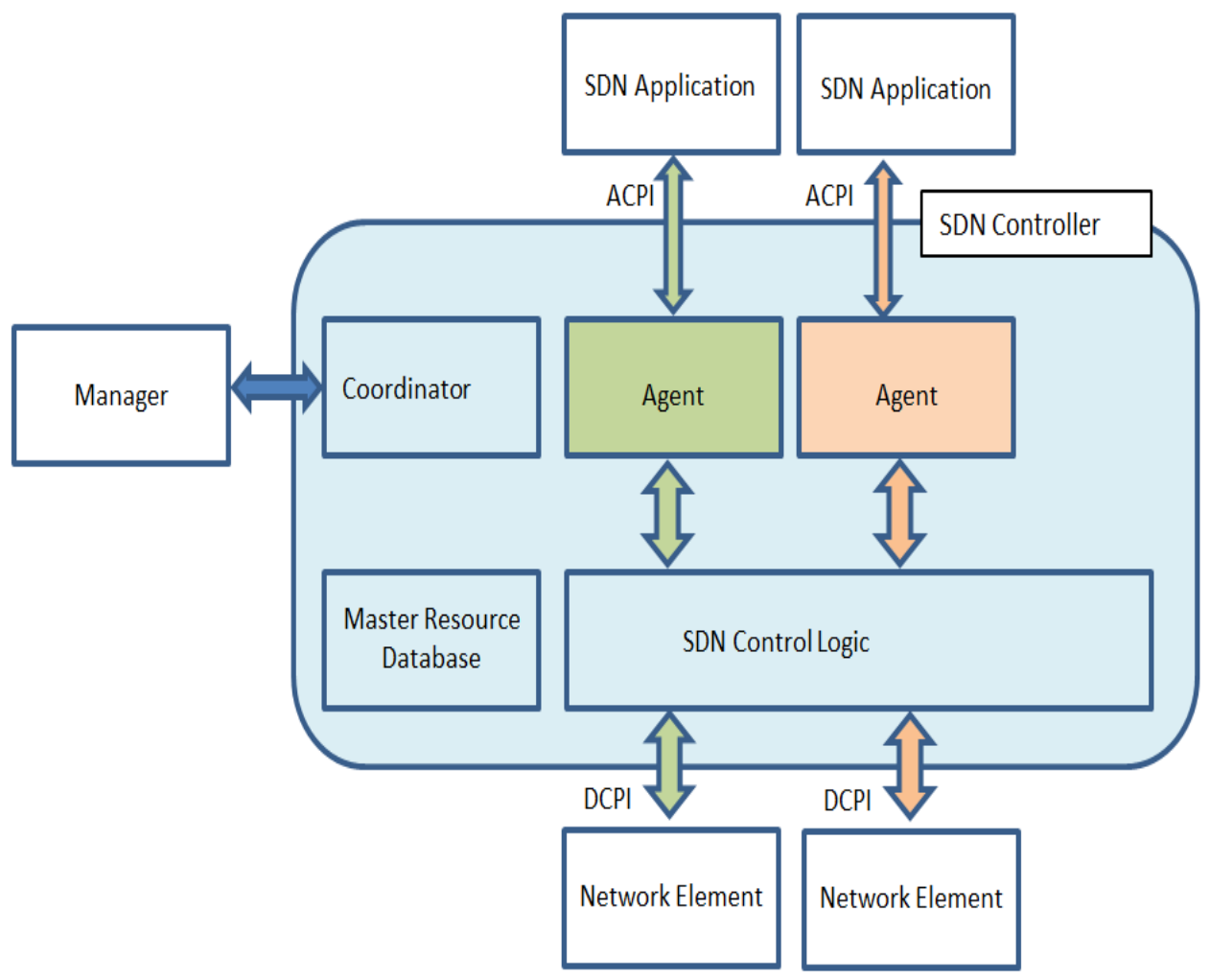

Figure 2. The Basic Architecture of an SDN Controller

The SDN controller directs the NEs on the data plane enabling them to optimize the flow mobility of multimedia traffic. It has the responsibility to administer the resources that are shared by multiple clients or applications. 


\section{Flow Mobility in Software Defined Networks}

The SDN enables applications to become network aware whereas the control and data planes are separated. The control plane provides a logically centralized mobility management while the multimedia traffic is forwarded through NEs in the data plane. The SDN controller simplifies the scheduling and resource allocation and has a complete control over the SDN datapaths for forwarding of multimedia traffic.

The multimedia traffic flow based on SDN architecture is shown in Figure 3. That is, Figure 3(a) indicates the multimedia traffic flow within the same domain, while the multimedia traffic flow in different domains is depicted in Figure 3(b). In Figure 3(a), the network element (i.e., $\mathrm{NE}_{6}$ ) detects the attachment of a mobile node (MN) and sends a control signal to the SDN Controller $\left(\mathrm{SDN}-\mathrm{C}_{1}\right)$ using and address update message. The $\mathrm{SDN}-\mathrm{C}_{1}$ then assigns a routable address to the $\mathrm{MN}$ and stores the mapping between the MN's routable address and its unique home address (HoA) into its master resource database (MRDB). The routable address has a similar functionality with the Care-of Address (CoA) in the centralized mobility management solutions.

The correspondent node $(\mathrm{CN})$ could be a multimedia service provider attached to the NE3 initializes a session with the MN. The NE3 queries the SDN-C1 with the MN's HoA as it is used as the destination address for the multimedia traffic intended for the MN. The SDN-C1 provides the NE3 with the complete optimized datapath with the MN's HoA as the destination address. The optimized datapath is computed by the SDN Controller as part of its functions. As soon as the datapath is downloaded, the multimedia traffic is then forwarded and traverses through the NEs indicated on the routing table (i.e., downloaded datapath).

In the case that MN changes its point of attachment (PoA), for example, from $\mathrm{NE}_{6}$ to $\mathrm{NE}_{1}$, the $\mathrm{NE}_{1}$ detects the MN's movement (obtains a new routing address) then sends an update message to $\mathrm{SDN}-\mathrm{C}_{1}$ informing the current movement of the $\mathrm{MN}$. The $\mathrm{SDN}_{-} \mathrm{C}_{1}$ updates the MN's routable address to its MRDB. Due to the intelligibility of $\mathrm{SDN}_{-} \mathrm{C}_{1}$, the session between $\mathrm{CN}$ and $\mathrm{MN}$ is already known, that means, it just need to rewrite the destination address, redirects the datapath, and continue forwarding the multimedia traffic to the MN's new routing address.

The flow mobility of multimedia traffic between different domains is shown in Figure 3(b). This scenario occurs whenever the MN changes its PoA from one network domain to another. Initially, $\mathrm{MN}$ is attached to $\mathrm{NE}_{6}$, thus $\mathrm{NE}_{6}$ is required to send an update message to the $\mathrm{SDN}-\mathrm{C}_{1}$ in order to update regarding the current routing address of the $\mathrm{MN}$ and the latter maps this routing address to the MN's HoA. In order to support adaptive SDN applications, the SDN controller are implemented in a distributed approach, enabling them to communicate and synchronize the mobility management of all NEs. This allows SDN controllers to share their intelligibility of the ongoing sessions among the NEs and communicating devices able to move around its domains. 


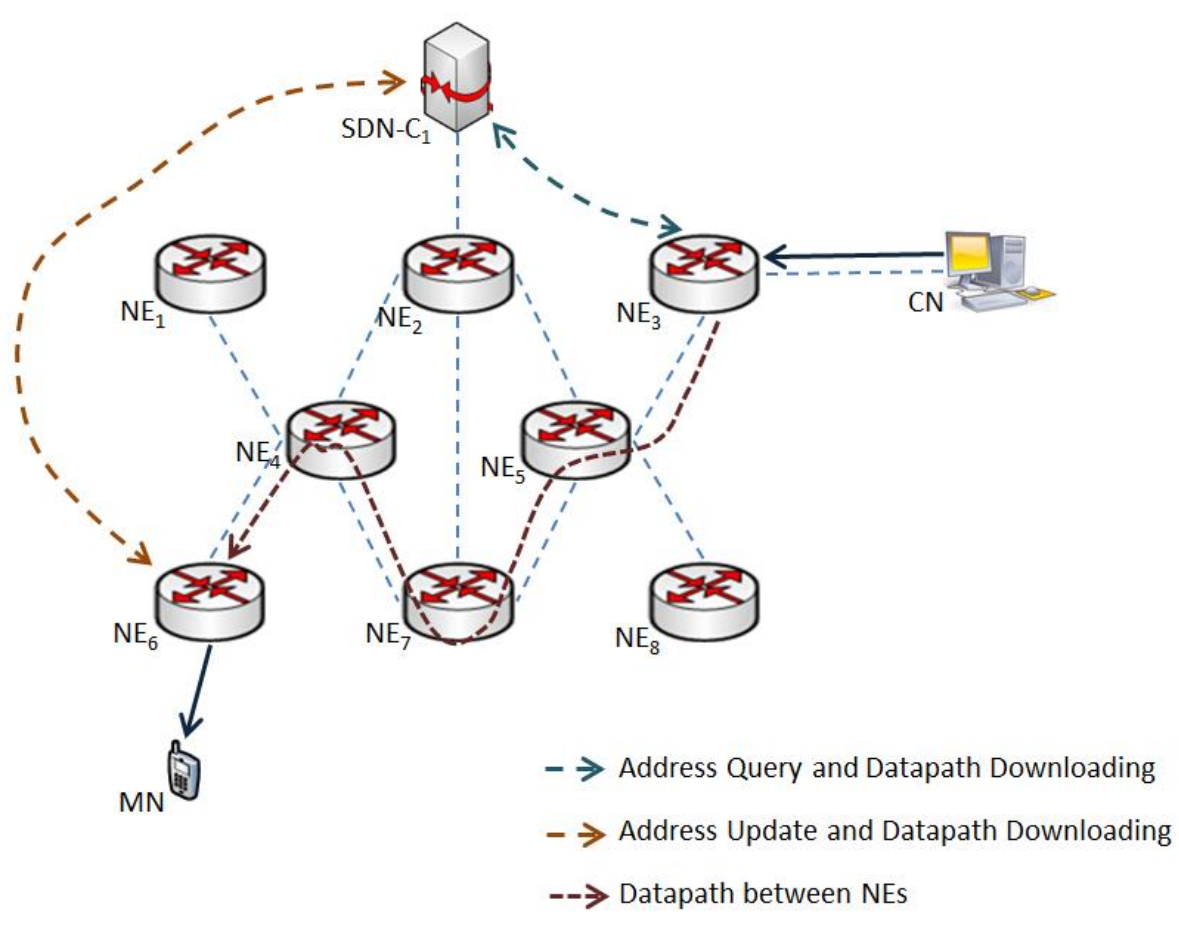

(a) Multimedia Traffic Flow based on SDN Architecture within the same Domain

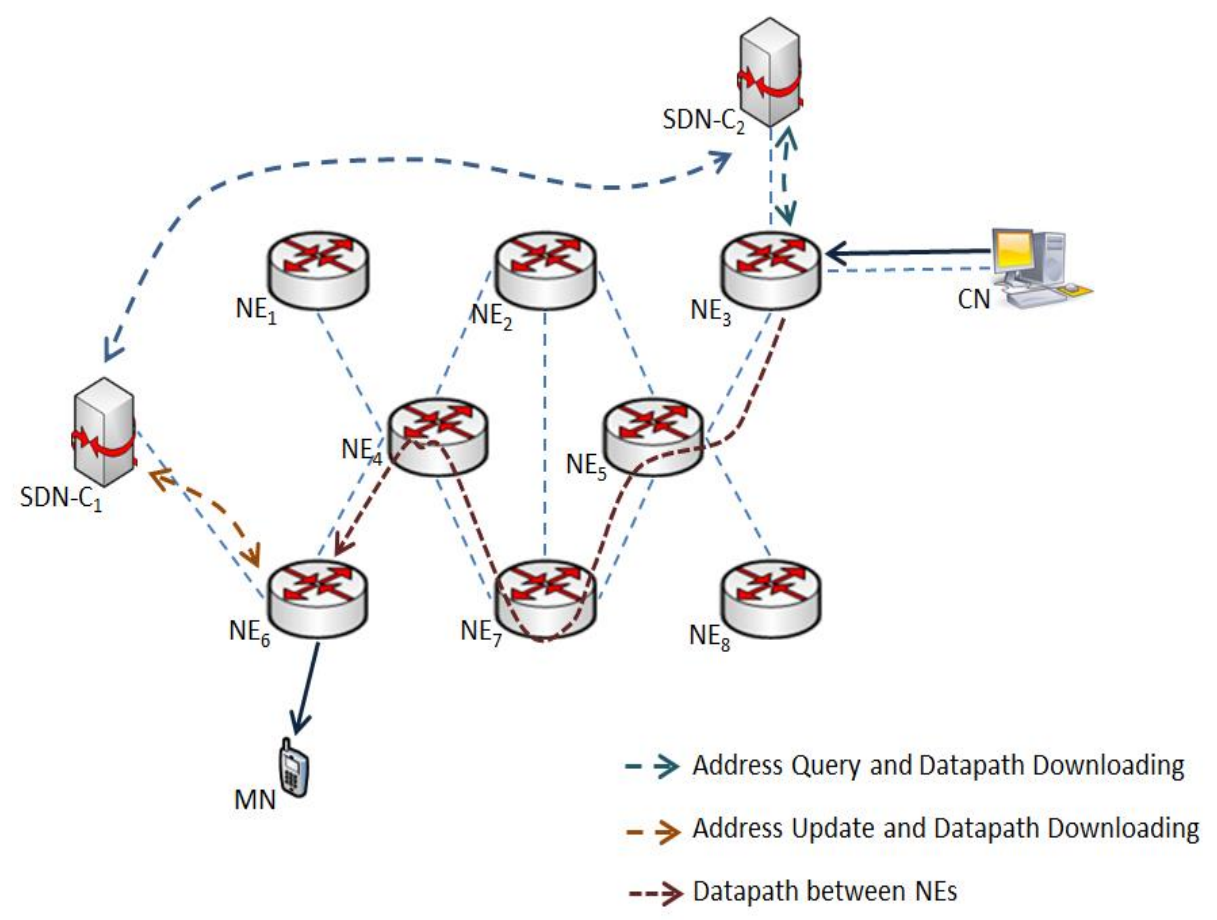

(b) Multimedia Traffic Flow based on SDN Architecture with different Domain

Figure 3. Multimedia Traffic Flow based on SDN Architecture

The SDN-C $C_{1}$ can query and forward the information on the MN to $\mathrm{SDN}-\mathrm{C}_{2}$ in order to calculate the most optimized route of multimedia traffic that the $\mathrm{CN}$ intends to forward to the MN. Thus, $\mathrm{SDN}-\mathrm{C}_{2}$ is able to provide the MN's routing address and datapath to $\mathrm{NE}_{3}$ to which the $\mathrm{CN}$ is currently attached. Whenever the MN moves 
outside the domain of SDN-C $\mathrm{C}_{1}$ and enter the $\mathrm{SDN}-\mathrm{C}_{2}$ domain, for example, the MN attaches to $\mathrm{NE}_{8}$, the $\mathrm{NE}_{8}$ send an update message to the $\mathrm{SDN}-\mathrm{C}_{2}$ in order to inform the MN's movement and updates the information on its MRDB. Multimedia traffic forwarding is resumed whenever necessary datapath information has been shared by both SDN controllers and provided it to the specific NEs involved in the routing table.

The SDN addresses the static performance of traditional network architectures. The use of multiple SDN controllers can make the mobility management become distributed enabling the SDN applications become adaptive. The separation of the control and data planes enabled a more efficient management, provisioning, and configuration, and orchestration of network resources.

\section{Conclusion}

This paper has analyzed the flow mobility of multimedia traffic across software defined networks. It has presented an implementation of SDN to direct the flow of multimedia traffic both in the same network domain and between different network domains of heterogeneous wireless networks. The implementation of multiple SDN controllers is essentially important to provide flexibility and dynamic network configurations and orchestration and enables the SDN applications to become adaptive. The analysis has implied that the handover between access networks becomes more efficient and multimedia traffic forwarding between mobile devices is optimized in a heterogeneous wireless environment.

\section{Acknowledgments}

This paper has been supported by the 2017 Hannam University Research Fund.

\section{References}

[1] CISCO, "Cisco Visual Networking Index: Forecast and Methodology, 2016-2021", White Paper, https://www.cisco.com/c/en/us/solutions/collateral/service-provider/visual-networking-indexvni/complete-white-paper-c11-481360.html, (2017) September.

[2] H. Chan, D. Liu, P. Seite, H. Yokota, J. Korhonen, "Requirements of Distributed Mobility Management", Internet Engineering Task Force, Request for Comments (RFC) 7333, (2014) August.

[3] P. Bertin, S. Bonjour, J. Bonnin, "Distributed of centralized mobility", In Proceedings of IEEE Global Telecommunications Conference (GLOBECOM 2009), IEEE, (2009), pp. 1-6.

[4] "Big Data: What it is and why it matter", https://www.sas.com/en_us/insights/big-data/what-is-bigdata.html, Retrieved: (2017) July 26.

[5] J. Strickland, "How Cloud Computing Works", HowStuffWorks, https://computer.howstuffworks.com/cloud-computing/cloud-computing.htm, Retrieved: (2017) July 30.

[6] "Software-Defined Networking (SDN) Definition", https://www.opennetworking.org/sdn-definition/, Retrieved: (2017) July 26.

[7] ONF White Paper, "Software-Defined Networking: The New Norm for Networks", (2012) April 13, https://www.opennetworking.org/images/stories/downloads/sdn-resources/white-papers/wp-sdnnewnorm.pdf.

[8] ONF White Paper, "SDN Architecture Overview", (2014) June 12, https://www.opennetworking.org/wpcontent/uploads/2013/02/TR_SDN_ARCH_1.0_06062014.pdf. 